\title{
Dynamically softened substrate regulates malignancy of breast tumor cells
}

\author{
$\mathrm{Mi} \mathrm{Hu}{ }^{1 \dagger}, \mathrm{Xu} \mathrm{Li}^{1 \dagger}$, Wei-Pin Huang ${ }^{1}$, Deng-Feng $\mathrm{Hu}^{1}$, Ke-Feng Ren ${ }^{1,2^{*}}$ and Jian $\mathrm{Ji}^{1,2}$
}

\begin{abstract}
It has long been hypothesized that an increase in the extracellular matrix (ECM) stiffness mechanoactivates malignant phenotypes of breast tumor cells by regulating an array of processes underlying cancer biology. Although the contribution of substrate stiffening to drive malignant phenotype traits and other biological functions of a tumor is increasingly understood, the functional role of substrate softening on breast cancer cellular responses has rarely been investigated. Herein, we employed matrix metalloproteinase (MMP)-sensitive film to perform assays to explore the consequences of lowering stiffness on the biological behaviors of breast cancer cell MDA-MB-231. We demonstrated that cells underwent dramatic changes in migration, cellular conjunction, and expression of malignance-associated proteins and genes when the substrate stiffness decreased. Based on RNA sequencing and analysis, we found that hub genes including TP53, CCND1, MYC, CTNNB1, and YAP were validated to play central parts in regulating stiffness-dependent cellular manner change. Moreover, through visualization of differentially expressed genes (DEGs), cells on dynamically softened substrate appeared less influenced by transfer to tissue culture polystyrene (TCPS). These results suggest substrates with decreasing stiffness could normalize breast tumor malignant phenotype and help cells store the mechanical memory of the consequential weaker malignance.
\end{abstract}

Keywords: substrate stiffness, dynamic softening, breast tumor cell, malignance normalization

\section{INTRODUCTION}

The extracellular matrix (ECM) stiffness in tumors often differs from that in corresponding normal tissues, exhibiting increased integrin clustering, focal adhesion ki- nase (FAK) activation and focal adhesion formation, and intensifying relevant cellular signaling pathways $[1,2]$. This alteration in tissue stiffness has been implicated in breast tumor development and progression, and mammography or manual palpation to identify regions of dense tissue is commonly used as early detection methods for breast cancer [3-5]. It has long been hypothesized that ECM stiffening mechanoactivates malignant phenotypes of breast tumor cells, including losing apical-basal polarity and adherent junctions, acquiring the ability to migrate and invading through the basement membrane $[6,7]$, collectively dysregulating breast tumor metabolism and promoting tumor growth and aggressiveness. In a recent study, researchers fabricated methacrylated hyaluronic acid (HA) hydrogels whose stiffness can be dynamically modulated by two-stage polymerization to mimic the gradual matrix stiffening during breast cancer development [8]. Mammary epithelial cells were found to lose epithelial characteristics and spheroid structure when the hydrogel stiffness increased from $<150$ to $>3000 \mathrm{~Pa}$. Other in vitro and in vivo studies have demonstrated that increasing matrix stiffness induces mechanomediator release from cytoplasmic anchor to enter the nucleus, and drive coordinate transcriptional events of epithelial-mesenchymal transition (EMT) and breast tumor invasion and metastasis [7]. Breast cancer cells undergo significant alterations in pathways relevant to metabolism when removed from soft host environment to rigid surfaces, and thereby showing dramatic phenotypic changes and drug responses $[9,10]$. Stiff matrices regulate prolactin signals and mammary epithelial morphogenesis by integrin clustering from physiologic to pro-tumorigenic outcomes, thereby perturbing adherent junctions, cell polarity, tu-

\footnotetext{
${ }^{1}$ MOE Key Laboratory of Macromolecular Synthesis and Functionalization, Department of Polymer Science and Engineering, Zhejiang University, Hangzhou 310027, China

${ }^{2}$ Key Laboratory of Cardiovascular Intervention and Regenerative Medicine of Zhejiang Province, Sir Run Run Shaw Hospital, Zhejiang University, Hangzhou 310016, China

† These authors contributed equally to this work.

* Corresponding author (email: renkf@zju.edu.cn)
} 
mor growth, and lumen formation [11,12].

Despite the connection between matrix stiffening and malignant outcomes in breast cancer cells has been largely shown, little is known about how lowering of substrate stiffness might induce the decrease of tumor cell malignance. Nearly 50 years ago, it was reported that carcinoma cells exhibit a tendency to differentiate to normal cells when co-cultured with embryonic mammary mesenchyme, which is assumed due to the production of critical molecular morphogens and alteration in gene activities [13,14]. Many following studies suggested that breast cancer cell lines can be normalized to more benign phenotypes in conjunction with inductive ECM components, as indicated by growth suppression, morphology switch, and histodifferentiation enhancement $[15,16]$. Biglycan, which is a small leucine-rich proteoglycan, was shown to influence tumor cell behavior and induce breast cancer normalization, and biglycan-induced differences in ECM mechanical property were suspected to play a key role in cancer normalizing effects [15]. Given another in vivo study showing collagen crosslinking and ECM stiffening can promote breast cancer formation by driving focal adhesion assembly and enhancing PI3K signaling while reduction of collagen crosslinking can inhibit tumor progression [17], it is possible that a decrease in ECM stiffness can reverse the malignant phenotype of tumor cells. However, these findings, while provocative, have not yet identified a link between lowering of matrix stiffness and more benign phenotype of malignant tumor cells.

Herein, we selected MDA-MB-231, one of the most commonly used triple-negative cell lines in metastatic breast cancer research, to perform assays for exploring consequences of lowering stiffness on the migration, cellular junction, relevant protein, and RNA expression, as well as the mechanical memory of normalized tumor cells. To achieve this, matrix metalloproteinase (MMP)sensitive peptide-crosslinked polyelectrolyte multilayered films whose stiffness can be dynamically decreased during cell culture, were employed. Compared with cells on static high-stiffness substrates, MDA-MB-231 cultured on substrates with dynamically lowering stiffness underwent dramatic changes in migration, cell-cell adhesion, expression of malignance-associated proteins ( $\beta 1$ integrin, E-cadherin, cadherin 11, MMP 2, MMP 9), and genes (EGFR, SOX4, TNC). Based on the Kyoto Encyclopedia of Genes and Genomes (KEGG) enrichment and proteinprotein interaction (PPI) network analysis, cell cycle, hippo signaling pathways, and hub node genes including TP53, CCND1, MYC, CTNNB1, and YAP were assumed to play a central part in regulating the stiffness-dependent cellular manner change. Moreover, less differentially expressed genes (DEGs), activated KEGG pathways, and expressed oncogenes were found between cells cultured on softening substrates before and after transfer to rigid TCPS, indicating normalized tumor cells by the decreasing stiffness possess the mechanical memory of the consequential weaker malignance.

\section{EXPERIMENTAL SECTION}

\section{Materials}

HA (sodium salt, $M_{\mathrm{w}} 351-600 \mathrm{kDa}$ ) was purchased from Lifecore (Oklahoma, USA). Poly-L-lysine (PLL, $M_{\mathrm{w}}$ 30,000-70,000), Methacrylate anhydride (AMA), N-dimethylformamide (DMF), Irgacure 2959 (I2959), 1-(3dimethylaminopropyl)-3-ethylcarbodiimide hydrochloride (EDC), and $N$-hydroxysulfo-succinimide sodium salt (sulfo-NHS) were purchased from Sigma-Aldrich (Hangzhou, China). MMP-sensitive peptide (GCRDGPQGIWGQ-DRCG) was purchased from Top Biotech (Shanghai, China). $N$-[2-hydroxyethyl]-piperazine- $N^{\prime}$-[2ethanesulfonic acid] (HEPES, free acid, high purity grade), phosphate-buffered saline (PBS), bovine serum albumin (BSA), and tris-buffered saline (TBS) were purchased from Sangon Biotech (Shanghai, China). All solutions were prepared by using deionized water $(18 \mathrm{M} \Omega$, Milli-Q Ultrapure Water System, Millipore).

\section{Fabrication of Stiff-to-Soft/Stiff polyelectrolyte multilayer films}

The Stiff-to-Soft/Stiff polyelectrolyte multilayers were constructed according to the methods described previously $[18,19]$. Glass coverslips (diameter: $14 \mathrm{~mm}$ ) were pretreated by immersion in piranha solution $\left(30 \% \mathrm{H}_{2} \mathrm{O}_{2} /\right.$ $\left.98 \% \mathrm{H}_{2} \mathrm{SO}_{4}=3 / 7, v / v\right)$ for $30 \mathrm{~min}$, and then cleaned by deionized water thoroughly. PLL $\left(0.5 \mathrm{mg} \mathrm{mL}^{-1}\right)$, and HA $\left(1 \mathrm{mg} \mathrm{mL}^{-1}\right)$ were dissolved in HEPES/ $\mathrm{NaCl}$ buffer solution (20 mmol L ${ }^{-1}$ HEPES, $0.15 \mathrm{~mol} \mathrm{~L}^{-1} \mathrm{NaCl}, \mathrm{pH}$ 7.4). The multilayers were fabricated by alternate deposition of glass coverslips into PLL and HA solutions for $8 \mathrm{~min}$ each. Before each step, $\mathrm{NaCl}$ solution $\left(150 \mathrm{mmol} \mathrm{L}^{-1}, \mathrm{pH}\right.$ 6.4) was used as wash liquor to flush the remnant molecules on the substrates. This sequence was repeated until 12 double-layered films were finished. To prepare Stiff-to-Soft films, (PLL/HA) ${ }_{12}$ films were immersed in a solution of MMP-sensitive peptide, GCRD-GPQGIWGQDRCG $\left(8 \mathrm{mg} \mathrm{mL}^{-1}\right.$, HEPES-NaCl buffer solution at $\mathrm{pH}$ 8.5) containing photoinitiator I2959 $\left(2 \mathrm{mg} \mathrm{mL}^{-1}\right)$ overnight. After that, the UV explosure at $365 \mathrm{~nm}$ was applied 
for $20 \mathrm{~min}$ to enhance the crosslinking reaction, and the films were then rinsed with HEPES/ $\mathrm{NaCl}$ buffer solution for at least eight times. To prepare Stiff films, (PLL/HA) 12 films were crosslinked for $18 \mathrm{~h}$ at $4^{\circ} \mathrm{C}$ with a solution containing EDC $\left(150 \mathrm{mg} \mathrm{mL}^{-1}\right)$ and sulfo-NHS $\left(11 \mathrm{mg} \mathrm{mL}^{-1}\right)$ in $\mathrm{NaCl}\left(150 \mathrm{mmol} \mathrm{L}^{-1}, \mathrm{pH}\right.$ 5.5). Similarly, the films were cleaned by rinsing with $\mathrm{HEPES} / \mathrm{NaCl}$ buffer solution for at least eight times.

\section{Breast tumor cell MDA-MB-231 culture assay}

MDA-MB-231 cells were cultured in DMEM supplemented with $10 \% \mathrm{FBS}$ at $37^{\circ} \mathrm{C}$ and $5 \% \mathrm{CO}_{2}$. The culture medium was changed every 2-3 days and cells with $90 \%$ confluence were passaged or seeded for further experiments. For the cell adhesion and proliferation experiments, the glass slides with multilayers were placed into 24-well plates. Cells were seeded on the samples at a density of $4 \times 10^{4}-6 \times 10^{4}$ per well for routine culture and $1 \times 10^{4}-2 \times 10^{4}$ per well for low-density culture. After 6 -h adhesion or 3-d proliferation, cells were fixed with $4 \%$ paraformaldehyde in PBS and then stained with FITCphalloidin (1:500, Sigma, USA), $\beta$-catenin (1:100, Sigma, USA), integrin- $\beta 4$ ( $1: 150$, Sigma, USA), and DAPI ( $1: 100$, Sigma, USA). All samples were mounted onto clean coverslips with antifade reagent (Invitrogen) and observed by fluorescence microscopy (Axiovert 200M, Zeiss, Germany). For the cell migration experiment, the samples were lined out a space with a tweezer after proliferation. Cell migration was observed and photographed each day for three days using phase-contrast microscopy (Axiovert 200M, Zeiss, Germany). Cell numbers were calculated by Image J software. For culture in matrigel, cells were isolated from the substrates by tyrisin and mixed with matrigel solution with a volume ratio of 1:4. The mixture was injected into 24 -well plates and cultured at $37^{\circ} \mathrm{C}$ and $5 \%$ $\mathrm{CO}_{2}$ for three days before immunofluorescence staining.

\section{Western blot analysis}

For western blotting assay, the samples were isolated by radio immunoprecipitation assay (RIPA) lysis buffer with protease inhibitors. The lysates were centrifuged at $12,000 \mathrm{r} \mathrm{min}^{-1}$ at $4^{\circ} \mathrm{C}$ for $15 \mathrm{~min}$ and separated on sodium dodecyl sulfate (SDS)-polyacrylamide gels. After being transferred to a poly(vinylidene difluoride) (PVDF) membrane (Millipore, MA, USA), the proteins were incubated overnight with antibodies against $\beta$-integrin, Ecadherin, cadherin 11, MMP 2, MMP 9, and $\beta$-actin. Membranes were then incubated with secondary antibody (Thermo Pierce). Finally, signals were detected using the ECL system (ECL Western Blotting Substrate, Pierce,
USA) and quantitated by densitometry using BandScan 5.0 software.

\section{Real-time quantitative PCR analysis}

Expression of MDA-MB-231 malignance relative genes was analyzed by real-time quantitative PCR (RT-qPCR). Briefly, cells were seeded in 6-well plates. RNA was extracted using a TRIzol Reagent kit (Haogene Biotech, China). The extracted and purified RNA samples (500 ng) were reversely transcribed into cDNA using a 1st-Strand cDNA Synthesis Kit (Haogene Biotech, China). Generated cDNA samples were used as templates to perform a standard PCR analysis using Power SYBR Master Mix (Invitrogen). PCR primers were designed to amplify human genes and PCR products were detected by real-time PCR detection systems (CFX384, Bio-Rad, USA).

\section{RNA-seq analysis}

Total RNA was extracted from MDA-MB-231 cells using TRIzol reagent (Invitrogen). Later, all the samples were sent to BGI Corporation (Shenzhen, China) for further RNA-seq detection and analysis via BGISEQ-500 sequencer. FPKM (fragments per kilobase of exon per million fragments mapped) was used to measure the gene expression in RNA-seq and DEGs were screened using Poisson distribution as in previous protocol [20]. The data were analyzed on the Dr. Tom network platform of BGI (http://report.bgi.com). The calculated $P$-values were then corrected by Bonferroni's method and a threshold of 0.05 was set. The KEGG database (http://www.genome.jp/ $\mathrm{kegg} / \mathrm{ko.html}$ ) was used for pathway mapping. Heatmap was plotted with pheatmap, and protein-protein associations were analyzed by STRING (https://string-db.org/) [21].

\section{RESULTS}

\section{Role of lowering substrate stiffness on adhesion and proliferation of breast tumor cells}

MMP-sensitive PLL and HA nanolayered films whose Young's moduli can be dynamically decreased from $(576.4 \pm 116.6)$ to $(180.5 \pm 74.0) \mathrm{kPa}$ in response to cellsecreted MMPs were employed [18] and hereafter denoted as Stiff-to-Soft. PLL/HA films covalently crosslinked by EDC whose stiffness is static-high were employed as the control substrate and denoted as Stiff. The Young's moduli of Stiff films were measured by the same technique with Stiff-to-Soft films and the value was (491 \pm 63$) \mathrm{kPa}$ [19], indicating the similar initial stiffness level of the two substrates. MDA-MB-231 cells were 
cultured on Stiff-to-Soft and Stiff films separately. As shown by the immunofluorescence images of cell adhesion on different substrates in Fig. 1a, b, after 18-h incubation, cell adhesion number and spread phenotype were practical unanimities. And after 5 -d proliferation (Fig. 1c, d), there was no significant difference in cell density and proliferation rate between Stiff-to-Soft and Stiff films $(P=0.5127)$. These results demonstrate that film systems with dynamically lowering stiffness guide spread phenotype and proliferation of breast tumor cells in a similar manner as the Stiff films.

\section{Role of lowering substrate stiffness on cell motility and cellular conjunction}

To investigate whether MDA-MB-231 cells exhibit different migration behaviors on substrates with different stiffnesses, we seeded the cells on the two types of films and applied a scratch migration test after cell proliferation (Fig. 2a, b). In fact, cells migrated at a lower percentage of metastatic cells on Stiff-to-Soft films $(7.91 \% \pm 0.82 \%)$, compared with cells on Stiff films, which migrated at a higher percentage of metastatic cells $(28.83 \% \pm 2.94 \%)$. Overall, these results indicate that the changes in matrix stiffness affected the migratory behavior of breast tumor cells, and the migration capacity of cells was significantly attenuated by stiffness decrease $(P=0.0024)$.

Since it has been reported that the migration and invasion of cancer cells depend on the disruption of cell-cell adhesion [22], we next assessed the influence of stiffness on the cellular conjunction of cells on the two different films. After the MDA-MB-231 cells were incubated with Stiff-to-Soft and Stiff in a low density for $18 \mathrm{~h}$, the immunofluorescent staining with $\beta$-catenin was conducted. $\beta$-Catenin directs tumor cell conjunction through adhesion molecular action and plays a central role in the Wnt signaling pathway which regulates cancer development and homeostasis [23]. Its translocation shifts from the cytoplasm to nuclear when cancerization occurs to participate in the transcription of multiple protooncogenes. According to Fig. 2c, cells cultured on Stiff-toSoft substrates showed a tendency to attach with each other, and high-expressed $\beta$-catenin (green) was observed in the interfaces of two connected cells. While on Stiff substrates, the cells dissociated from one another and showed weaker $\beta$-catenin expression. Furthermore, a three-dimensional (3D) matrigel culture model was developed to offer a biomimetic microenvironment to research the cellular behavior. Matrigel is a soluble matrix extracted from Engelbreth-Holm-Swarm murine, including laminin, collagen type IV, nidogen, heparin sulfate glycoprotein, and various cytokines, which could not only satisfy the need of visualization and controllability but also stimulate the growth habit and the structure of the cell in vivo. After MDA-MB-231 overgrown on Stiffto-Soft and Stiff, the cells were washed off by trypsin and moved to matrigel to observe their growth situation and
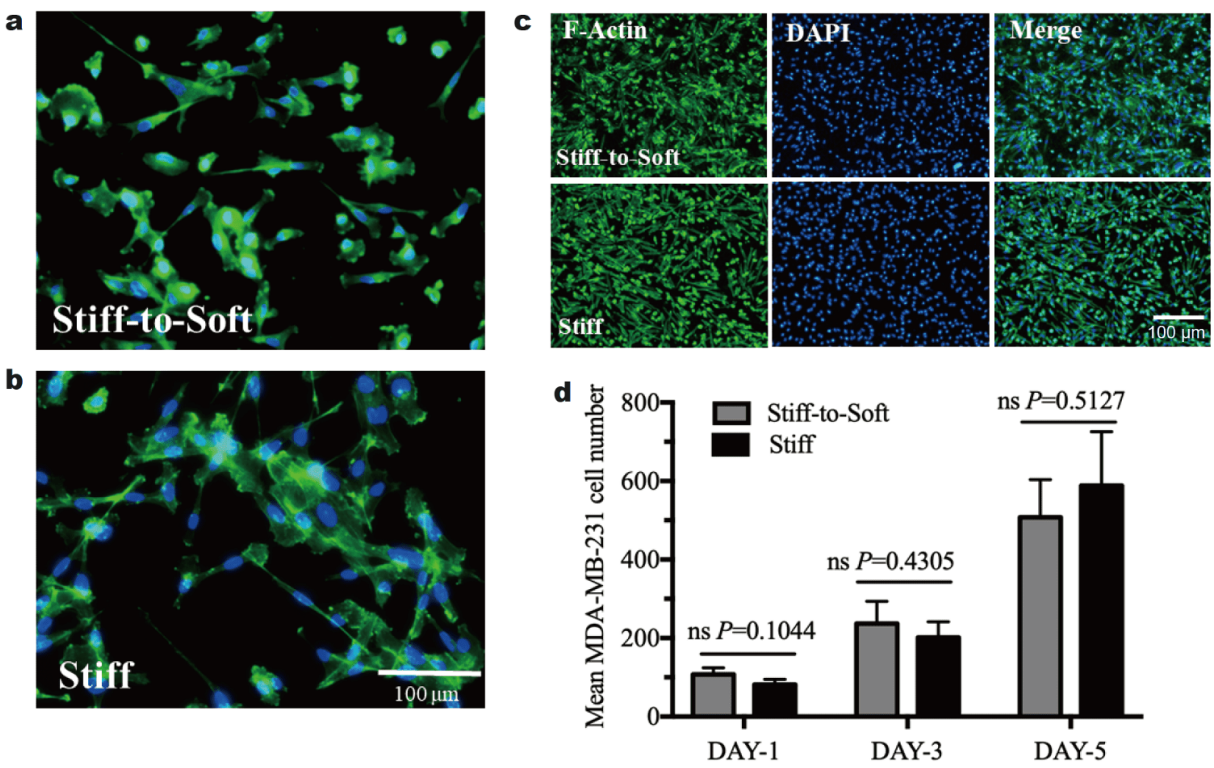

Figure 1 Stiff-to-Soft film guided cell spreading and proliferation in a similar manner as the Stiff films. (a-c) Immunofluorescent staining of cells for F-actin (green) and DAPI (blue) after 18-h adhesion (a, b) and 5-d proliferation (c) on Stiff-to-Soft and Stiff films. Scale bar $=100 \mu \mathrm{m}$. (d) Average cell numbers within the two substrates during proliferation (ns: not significant). 

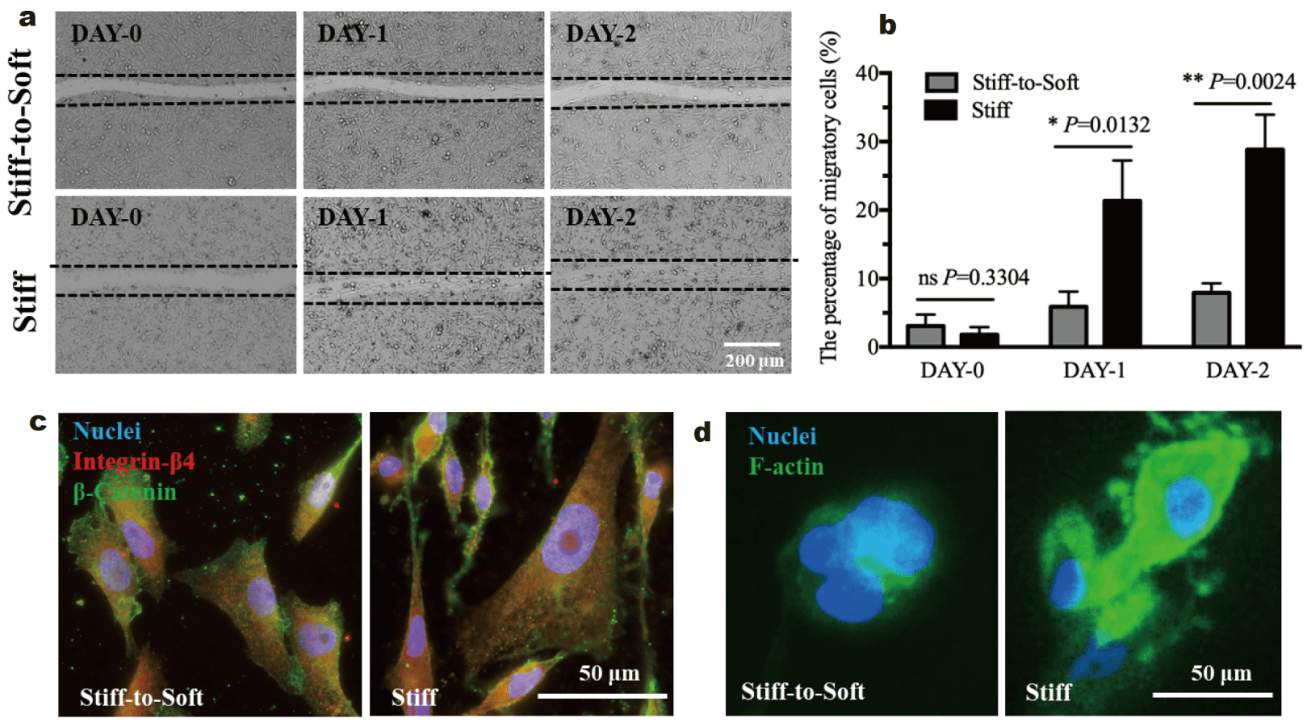

Figure 2 Role of dynamically lowering stiffness on breast tumor cell motility and cell-cell adhesion. (a) Scratch migration assay showing the different migration capacities of cells cultured on Stiff-to-Soft and Stiff films, respectively. Scale bar $=200 \mu \mathrm{m}$. (b) The percentage of migratory cells from Stiff-to-Soft after 1-2 d migration is significantly lower than that from Stiff. $* P<0.05, * * P<0.01$. (c) Immunofluorescent staining of cells for $\beta$ catenin (green), integrin- $\beta 4$ (red), and DAPI (blue) after low-density culture. Scale bar $=50 \mu \mathrm{m}$. (d) Immunofluorescent staining of cells in matrigel for F-actin (green) and DAPI (blue) after transfer from Stiff-to-Soft and Stiff. Scale bar $=50 \mu \mathrm{m}$.

cellular morphology. As shown in Fig. 2d, cells detached from Stiff-to-Soft preferred to huddle and form spherical clusters, while for the cells transferred to the matrigel after being cultured on Stiff, the formed cell clusters presented divergent structure. Consequently, these data suggested that breast cancer cells exhibited tighter cellcell conjunction and adhesion on matrices with lowering stiffness.

\section{Dependence of expression of malignancy-associated proteins or genes on substrate stiffness}

Cancer cells undergo fundamental alterations in the aspect of cell physiology to obtain a malignant phenotype. During this process, tumorigenic cells dissociate from their original tissue mass, reorganize their attachment with ECM through changes in adhesive dynamics, and degrade surrounding ECM to eventually invade to distant parts of the body [22]. Cell-cell adhesion proteins, such as E-cadherin, are completely or partially lost in advanced cancers and have been linked to a higher incidence of metastasis [22]. MMP 2 and MMP 9, which can degrade ECM and have a close relation to tumor invasion and metastasis, have been proved to exhibit high expression in patients with metastatic breast cancer [24-26]. To further clarify the mechanism of the influence of substrate stiffness on the cell malignancy of MDA-MB-231, we utilized western blotting to determine the expression of adhesion- associated proteins: $\beta 1$ integrin, E-cadherin, cadherin 11, and metastasis-associated proteins: MMP 2, MMP 9. Western blot data (Fig. 3a, b) indicated that compared with cells on Stiff, the dynamic decrease of stiffness promoted the expression of $\beta 1$ integrin, E-cadherin, and cadherin 11 by $39.7 \%, 33.4 \%$, and $28.0 \%$, respectively, and inhibited the expression of MMP 2 and MMP 9 by $53.0 \%$ and $65.7 \%$ respectively.

Next, we performed RT-PCR to evaluate the expression levels of EGFR, SOX4, and TNC in MDA-MB-231 cells on Stiff-to-Soft and Stiff films. EGFR is a receptor tyrosine kinase (RTK) involved in the regulation of cell growth and proliferation. Activation of EGFR stimulates tumor survival, progression, and migration, and overexpression of EGFR is frequently observed in the triplenegative subgroup of breast cancer $[27,28]$. SOX4, which is a developmental transcription factor in the ECM, has been widely shown to contribute to the metastatic spread of many solid cancer types including breast tumor $[29,30]$. Overexpression of SOX4 could inhibit breast cancer cell apoptosis and drive metastatic progression $[31,32]$. Tenascin C (TNC) is a hexameric glycoprotein that binds to fibronectin, integrin cell adhesion receptors, etc., and highly expressed in numerous human malignancies $[33,34]$. According to RT-PCR data (Fig. 3c), gene expression quantities of EGFR $(P=0.0008)$, SOX4 $(P=0.0541)$, and TNC $(P=0.0182)$ were significantly 

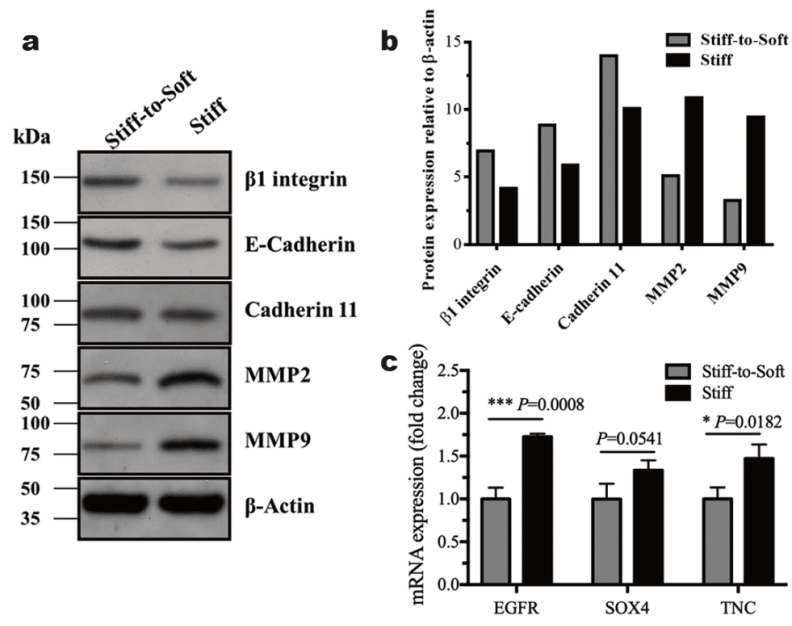

Figure 3 Dependence of the expression of malignancy-associated proteins and genes on the substrate stiffness. (a) Western blotting assay of adhesion-associated proteins: $\beta 1$ integrin, E-cadherin, cadherin 11 , and metastasis associated proteins: MMP 2, MMP 9. (b) Relative expression of proteins in western blotting images. (c) RT-PCR assay of malignancy-associated genes: EGFR, SOX4, and TNC. $* P<0.05, * * P<$ $0.01, * * * P<0.001$. down-regulated in cells on Stiff-to-Soft films compared with the cells on Stiff films. Thus, dynamically lowering stiffness was associated with decreased tumor malignancies through changing the adhesive and metastatic manners in MDA-MB-231 cell populations.

\section{Dependence of cell signaling pathway activation on substrate stiffness}

Through whole transcriptome RNA sequencing experiments, we next explored the stiffness-dependent alterations in cellular total gene expression to identify the role of substrate stiffness in the signaling pathways important to cell malignancy. Large differences in global gene expression were observed between cells cultured on Stiff-toSoft and Stiff substrates, with 6048 DEGs (using foldchange $\geq 2$ and $P$ value $<0.05$ ). The heatmap of the identified DEGs was shown in Fig. 4a. The impacts of substrate stiffness on the expression of top significantly affected genes implicated in adherent junction, cancer metastasis, cell cycle, and hippo pathway were examined (Fig. 4b-e, Table S1).
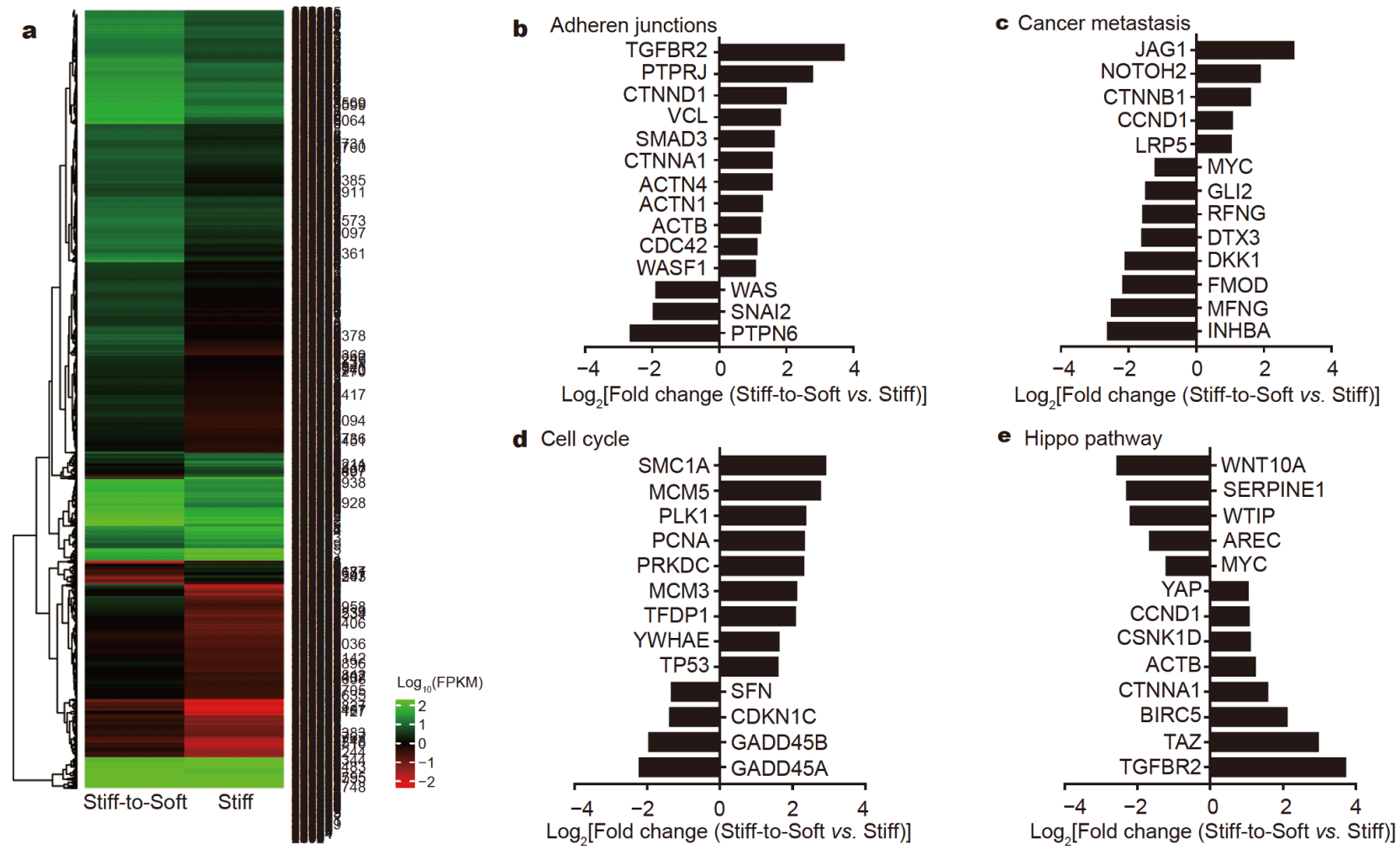

Figure 4 Substrate stiffness alters the expression of adhesion- and metastasis-regulatory genes. (a) Gross changes in cell gene expression when cultured on Stiff-to-Soft and Stiff, in unit of $\log _{10}$ (FPKM). Cutoffs were set at $\geq 2$ folds up-regulated (green) or $\leq-2$ folds down-regulated (red). (b-e) Fold changes in the expression of selected genes playing important roles in adherent junction (b), cancer metastasis (c), cell cycle (d), and hippo signaling (e). Foldchange is calculated by the formula: Foldchange $=$ (FPKM Stiff-to-Soft)/(FPKM Stiff). $P$ value cutoff is set at $P<0.05$. 
Analyzing the pathway annotations help to further interpret the biological functions of the differently expressed genes. The KEGG is a major public pathwayrelated data-base. To investigate the functions of the large range of gene signatures obtained, we performed KEGG pathway analysis, and the 6048 DEGs from Stiff-to-Soft $v s$. Stiff were summarized in six main categories including terms of cellular processes, environmental information processing, genetic information processing, human disease, metabolism, and organismal systems categories (Fig. 5a, Table S2). KEGG pathway enrichment indicates that DEGs were significantly enriched in 84 pathways and the top 20 pathways are shown in Fig. 5b, Table S3. The most two significant pathways were cell cycle and hippo signaling. For the DEGs selected in Fig. 4, PPI construction helped us understand the relationships of these gene expression differences, closely related to stiffness changes. We performed a PPI network by SRING database, a common online approach known to predict protein-protein interactions (Fig. 5c). Based on the results from the STRING database, hub node genes with high degrees including TP53, CCND1, MYC, CTNNB1 and YAP were identified, suggesting these genes may play an important part in the stiffness-dependent breast tumor biological alterations.

\section{Lowering stiffness help cells store mechanical memory of past environments}

A growing body of reports revealed that cells can remember past mechanical environments and this kind of memory influences long-term cellular fates, even after translocation into the body [35-37]. To determine whether or not breast tumor cells possess mechanical memory of weaker malignance on the substrate with lowering stiffness, we assayed DEGs in cells before and after transfer to rigid TCPS whose elastic modulus was at the GPa level. After transfering the cells from Stiff-to-Soft and Stiff substrates to TCPS for three days, we performed RNA-seq experiments and DEGs analysis. A total of 2928 DEGs were detected between cells before and after transfer to rigid TCPS from Stiff-to-Soft films. Among them, 231 genes were up-regulated and 2697 genes were down-regulated. A total of 6530 DEGs were detected between cells before and after transfer to rigid TCPS from Stiff films. Among them, 5441 genes were up-regulated and 1089 genes were down-regulated. The volcano plots of the DEGs are shown in Fig. 6a and b, suggesting that gene expression in breast tumor cells on Stiff-to-Soft films appeared less influenced by transfer to TCPS. Venn analysis for two comparisons emphasized 1756 over- lapping DEGs (Fig. 6c), suggesting the expression of these genes not only obviously changed in the Stiff group but more significantly changed in the Stiff-to-Soft group. These genes might participate in the specific molecular mechanisms of stiffness-dependent biological alterations.

KEGG pathway analysis was also performed to investigate the functions of the range of gene signatures obtained (Table S4). We obtained 22 significantly enriched pathways with DEGs detected between cells before and after transfer to rigid TCPS from Stiff-to-Soft films (Fig. 7a). The most significant pathway was proteoglycans in cancer. We obtained 89 significantly enriched pathways with DEGs detected between cells before and after transfer to rigid TCPS from Stiff films (Fig. 7b). The most significant pathway was the cell cycle. Furthermore, we examined the expression change of oncogenes and protooncogenes of breast cancer according to the COSMIC database. The data in Fig. $7 c$ are derived from an experiment where the genetic profile of cells cultured on Stiff-to-Soft and Stiff films as compared with those same cells transferred to TCPS substrates. Notably, after transfer to TCPS, members that encode for important oncogenes and proto-oncogenes in breast cancer including the epidermal growth factor receptor subfamily (ERBB2) were found to be widely down-regulated in cells previously grown on Stiff-to-Soft substrates as compared with Stiff substrates. The data above indicated that dynamically lowering stiffness helped breast tumor cells store the mechanical memory of the previous environments and the consequential weaker malignance was maintained even after cells were reverted to the rigid matrix.

\section{DISCUSSION}

In this study, we sought to better understand how matrix softening affects breast cancer cell malignant phenotype. Using an MMP-sensitive (PLL/HA) film system, we observed that dynamical changes in substrate stiffness are sufficient to modulate malignance in MDA-MB-231 cell populations. Compared with cells on static-high stiffness film (Stiff), when MDA-MB-231 were cultured on films whose stiffness can be dynamically lowered (Stiff-to-Soft), the migration capacity was significantly attenuated, and cell-cell conjunction was improved by stiffness decrease. These findings were further verified by comparison in the quantification of expression of malignancy-associated proteins and genes between cells on the two cultures. Cell cycle and hippo signaling pathways were proved to play the most important parts in the stiffness-dependent breast tumor biological changes, among which TP53, 


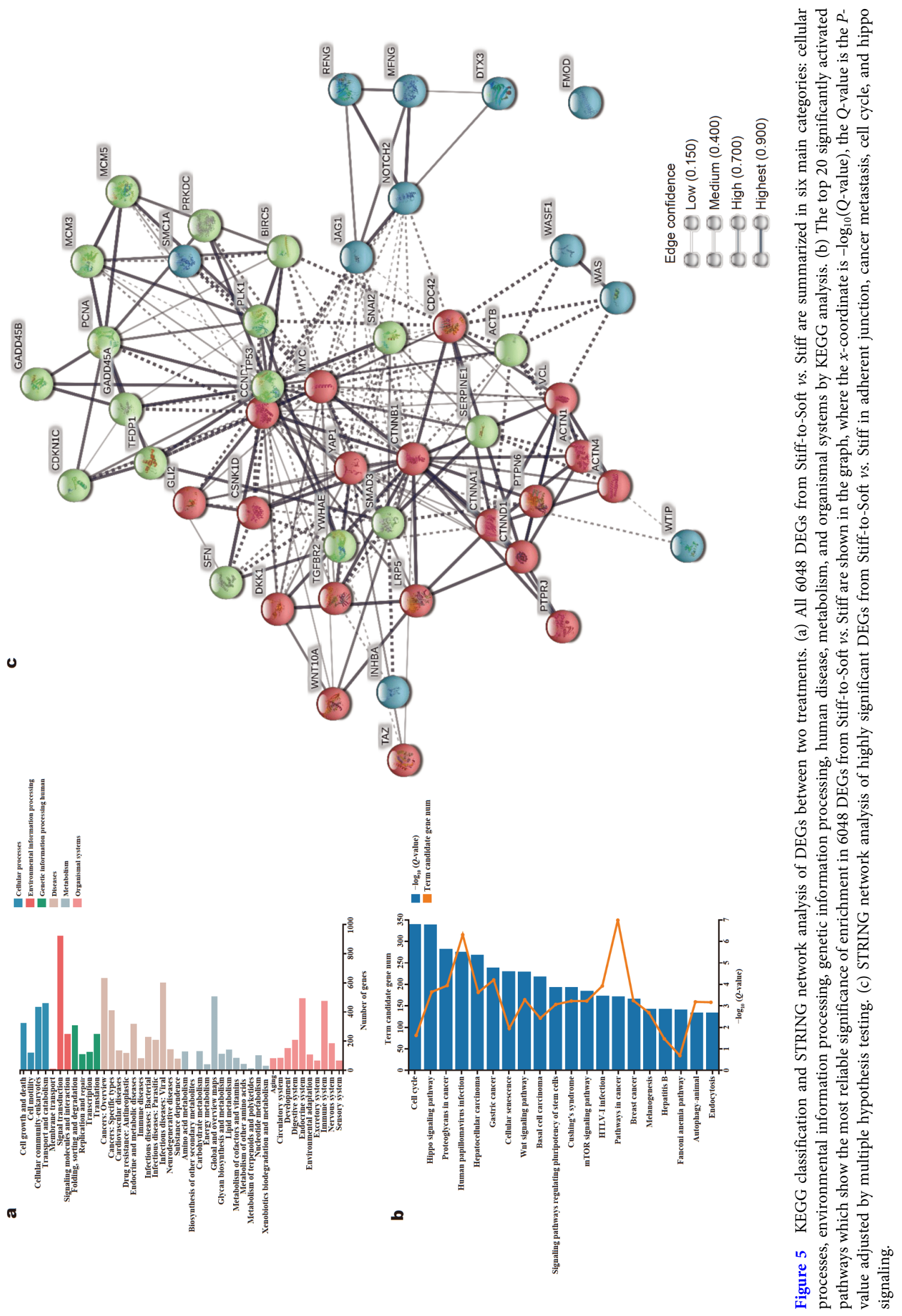



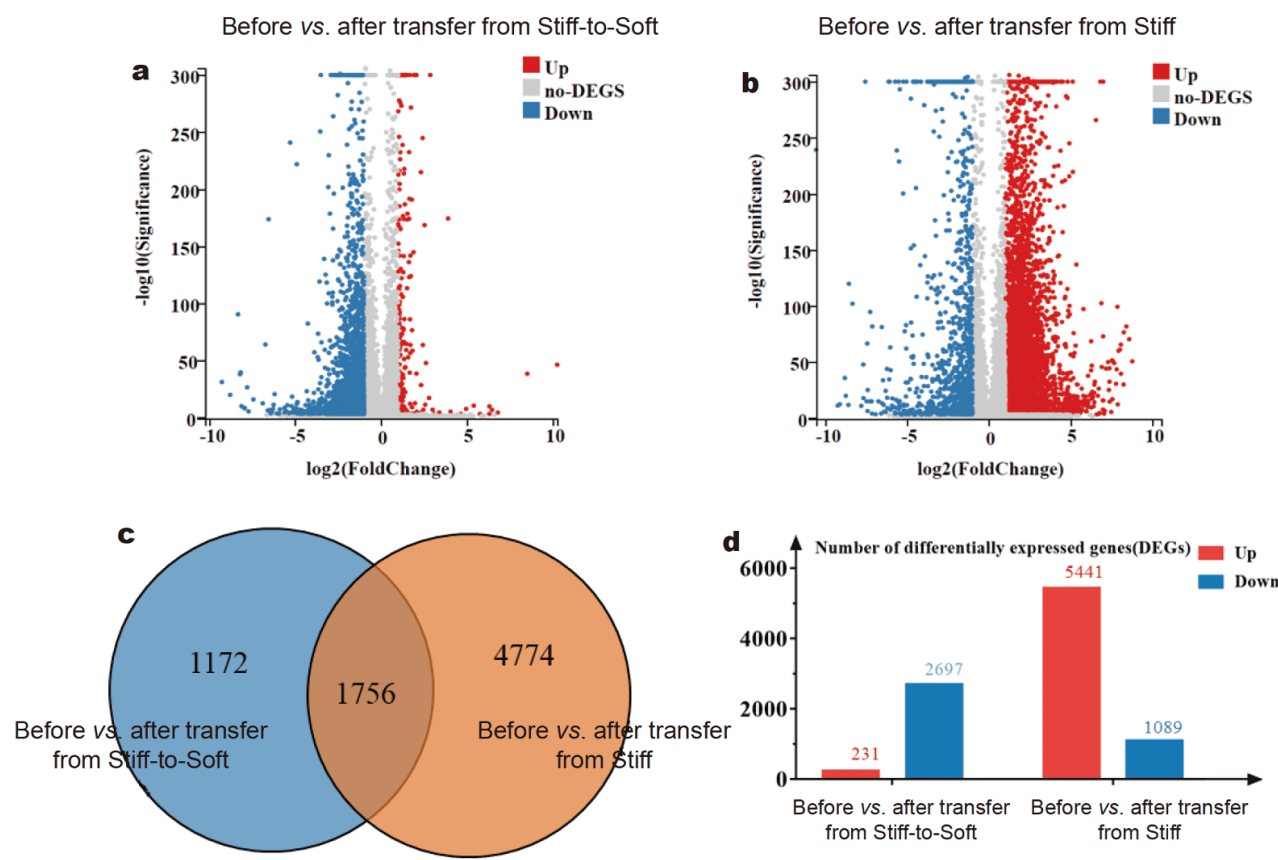

Figure 6 Visual representation of DEGs in cells between before and after transfer to rigid TCPS from Stiff-to-Soft or Stiff. (a, b) Volcano plots depict significantly up-regulated (red) and down-regulated (blue) DEGs before and after transfer to rigid TCPS from Stiff-to-Soft (a) or Stiff (b). Foldchange is calculated by the formula: Foldchange $=($ FPKM TCPS from Stiff-to-Soft $) /($ FPKM Stiff-to-Soft $)$ or Foldchange $=($ FPKM TCPS from Stiff $) /($ FPKM Stiff). FDR $\leq 0.001$, Foldchange $\geq 2$. (c) Venn diagram indicating the number of significant DEGs between the two comparisons. (d) Total numbers of genes up-regulated or down-regulated in the two groups are denoted.

CCND1, MYC, CTNNB1, and YAP were hub node genes. Notably, through analysis of DEGs in MDA-MB-231 before and after transfer to the rigid matrix, breast tumor cells normalized by decreasing stiffness are found to have the capacity of storing mechanical memory of past physical environments and weaker malignance.

Studies carried out previously suggested that cancers can differentiate and normalize their phenotypes when combined with embryonic inductive ECMs or interfered with adhesion molecules [38-40]. Mechanical property has been confirmed to be a critical control element in cells' switch between phenotypes central for neoplastic transformation including proliferation, differentiation, migration, and apoptosis by mechanically altering the cytoskeletal structure [14]. And in vivo inhibition of the activity of LOX, one class of ECM crosslinking enzymes, reduces collagen crosslinking, focal adhesions formation, and breast cancer progression [17]. These reports support our findings and highlight a role for stiffness in normalizing tumor malignance. Furthermore, the ECM surrounding cells undergo consistently dynamic remodeling and progressive stiffening/softening during tissue regeneration and disease progression [41,42]. Dynamic features of substrates especially the changes in stiffness significantly influence cellular behavior, function and the final fate determination $[42,43]$. Unlike the previous static disease modles regulating cell behaviors by certain stiffness, our MMP-responsive film dynamically decreases stiffness to regulate tumor cell malignance in a biomimetic way.

The cell cycle pathway consisting of DNA synthesis (S) and mitosis $(\mathrm{M})$ phase includes many processes necessary for self-replication and self-renewal. Cumulative clinical evidence shows deregulated cell cycle control might result in the aetiology of many human malignancies [44]. The hippo signaling pathway is a tumor suppressor regulatory pathway, playing an important role in regulating tissue size and tumorigenesis by inhibiting overgrowth and promoting apoptosis [45]. Since they are the most two significant pathways enriched by DEGs, it is assumed that dynamically lowering stiffness may correct some central aspects of cell signaling in the two pathways to normalize malignant phenotypes. TP53, CCND1, MYC, CTNNB1, and YAP are found to be key node genes in cell cycle and hippo signaling. TP53 is a key tumor suppressor that is relevant to cell migration and proliferation, and it is commonly mutated or deleted in various human cancers including breast cancer $[44,46,47]$. CCND1 mediates G1 


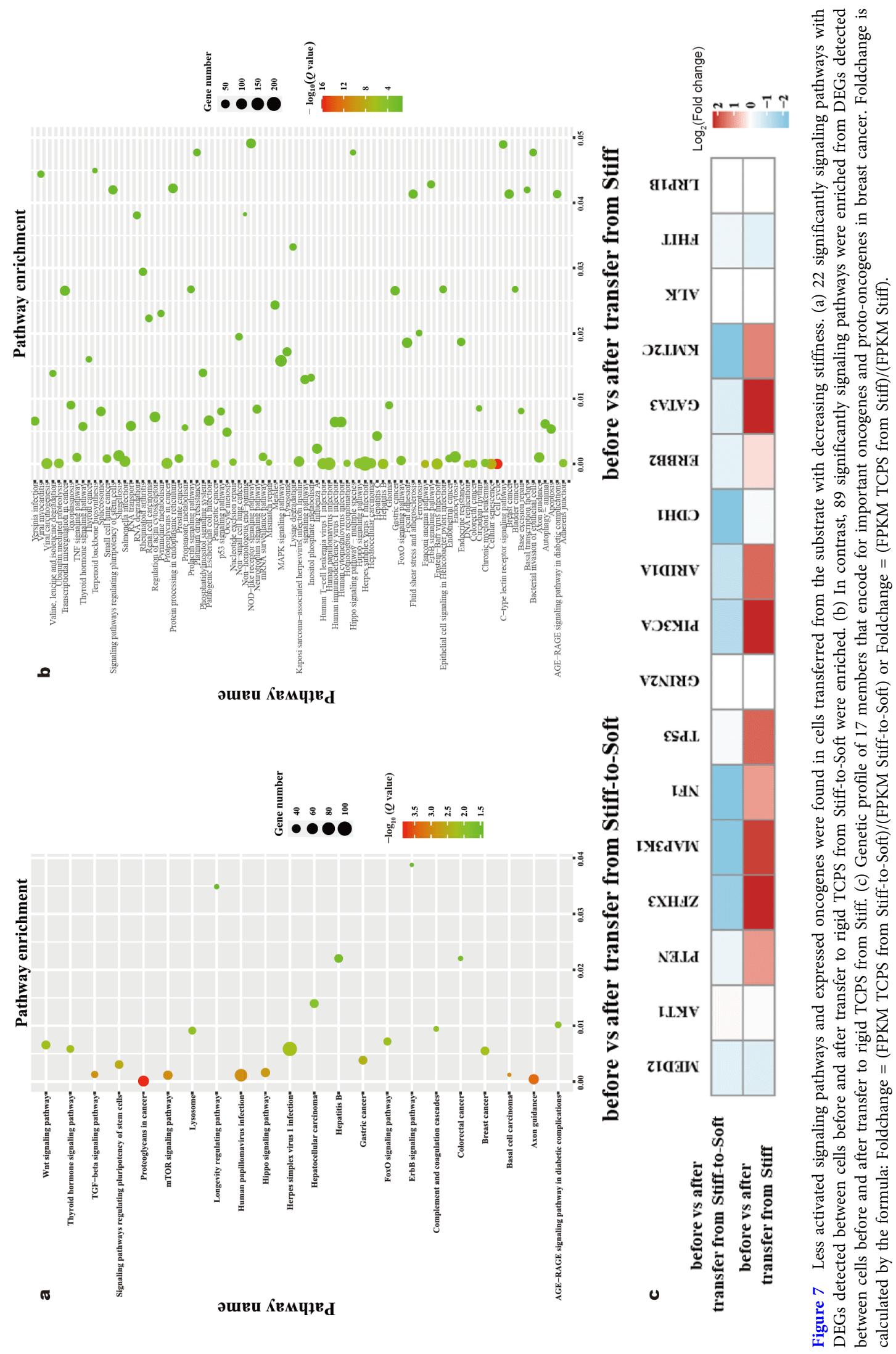


to $S$ phase transition in the cell cycle process, and overexpression of cyclin D1 has been linked to both better and worse malignance in breast cancer [48-50]. MYC, an essential signaling gene in multiple cellular processes including metabolism, metastasis, and stress pathway, has been widely reported to exhibit overexpression and activation in aggressive breast tumors [51]. Taken together, the up-regulation of TP53 and down-regulation of MYC on Stiff-to-Soft proved weakened tumor malignancy can be achieved by decreasing substrate stiffness. CTNNB1 gene that encodes $\beta$-catenin interacts with adhesion molecules and epigenetic regulators [52,53]. Its RNA sequencing experiment data are in consistence with the $\beta$ catenin immunofluorescent staining result, suggesting that cells present tighter cell-cell conjunction and adhesion on matrices with lowering stiffness.

YAP, a transcriptional regulator and downstream effector of the hippo signaling pathway, has been validated as a universal mechanotransducer [54,55]. Our finding that YAP expression in cells cultured on Stiff-to-Soft films is higher than that on Stiff films contrasts with previous work, in which it was found that increased 2D substrate stiffness promotes YAP accumulation in the nucleus and improved expression of YAP target genes [55-57]. Since several recent studies implicate cell signaling can be significantly changed by culture dimensionality and increased $3 \mathrm{D}$ culture and in vivo stiffness result in a lack of YAP activity [55-58], these indicate that there are multiple mechanisms by which cells sense and respond to environment stiffness, and the mechanism investigated in dynamic film systems might be more relevant to that in a $3 \mathrm{D}$ culture other than $2 \mathrm{D}$ culture. Furthermore, YAP-dependent mechanical memory which is defined as the capacity of cells to retain mechanical information from past physical environments has gained great attention recently [36,37]. YAP activation has been shown to enhance memory-dependent cellular responses while YAP-depletion blunts mechanical memory [37]. According to our data, cells on Stiff-to-Soft exhibit smaller differences before and after transfer to rigid TCPS, implying the phenomenon that dynamically lowering stiffness helps breast tumor cells store the mechanical memory of previous culture environments and the consequential weaker malignance might due to higher YAP expression.

\section{CONCLUSIONS}

In summary, this finding highlights the normalization effect of dynamically lowering stiffness of polyelectrolyte multilayers on migration, cell-cell adhesion, malignance relevant proteins ( $\beta 1$ integrin, E-cadherin, cadherin 11 , MMP 2, MMP 9), and genes (EGFR, SOX4, TNC) expression of breast cancer cell MDA-MB-231. Adding to the current paradigm that substrate stiffening mechanoactivates malignant phenotypes of the tumor, our work suggested substrate softening normalized cancer cells in turn through regulating migratory behavior and cell conjunction. Hub genes (TP53, CCND1, MYC, CTNNB1, and YAP) in cell cycle and hippo signaling pathways played important roles in mediating the effects of substrate stiffness on malignant plasticity. Furthermore, we demonstrated that decreasing stiffness promoted breast tumor cells to store the mechanical memory of the previous environments and the consequential weaker malignance was maintained eventhough the cells were reverted to the rigid matrix. Given the importance of mechanoregulation in cancer formation and progression, such information would have broad implications not only in biological responses but also in therapeutic treatments.

\section{Received 7 January 2021; accepted 1 February 2021;}

published online 30 April 2021

1 Chaudhuri O, Koshy ST, Branco da Cunha C, et al. Extracellular matrix stiffness and composition jointly regulate the induction of malignant phenotypes in mammary epithelium. Nat Mater, 2014, 13: $970-978$

2 Yu H, Mouw JK, Weaver VM. Forcing form and function: Biomechanical regulation of tumor evolution. Trends Cell Biol, 2011, 21: $47-56$

3 Acerbi I, Cassereau L, Dean I, et al. Human breast cancer invasion and aggression correlates with ECM stiffening and immune cell infiltration. Integrative Biol, 2015, 7: 1120-1134

4 Ishihara S, Inman DR, Li WJ, et al. Mechano-signal transduction in mesenchymal stem cells induces prosaposin secretion to drive the proliferation of breast cancer cells. Cancer Res, 2017, 77: 61796189

5 Lu P, Weaver VM, Werb Z. The extracellular matrix: A dynamic niche in cancer progression. J Cell Biol, 2012, 196: 395-406

6 Wei SC, Yang J. Forcing through tumor metastasis: the interplay between tissue rigidity and epithelial-mesenchymal transition. Trends Cell Biol, 2016, 26: 111-120

7 Wei SC, Fattet L, Tsai JH, et al. Matrix stiffness drives epithelialmesenchymal transition and tumour metastasis through a TWIST1-G3BP2 mechanotransduction pathway. Nat Cell Biol, 2015, 17: 678-688

8 Ondeck MG, Kumar A, Placone JK, et al. Dynamically stiffened matrix promotes malignant transformation of mammary epithelial cells via collective mechanical signaling. Proc Natl Acad Sci USA, 2019, 116: 3502-3507

9 Medina SH, Bush B, Cam M, et al. Identification of a mechanogenetic link between substrate stiffness and chemotherapeutic response in breast cancer. Biomaterials, 2019, 202: 1-11

10 Mah EJ, Lefebvre AEYT, McGahey GE, et al. Collagen density modulates triple-negative breast cancer cell metabolism through adhesion-mediated contractility. Sci Rep, 2018, 8: 17094 
11 Paszek MJ, Zahir N, Johnson KR, et al. Tensional homeostasis and the malignant phenotype. Cancer Cell, 2005, 8: 241-254

12 Barcus CE, Keely PJ, Eliceiri KW, et al. Stiff collagen matrices increase tumorigenic prolactin signaling in breast cancer cells. J Biol Chem, 2013, 288: 12722-12732

13 DeCosse JJ, Gossens CL, Kuzma JF, et al. Breast cancer: Induction of differentiation by embryonic tissue. Science, 1973, 181: 10571058

14 Ingber DE. Can cancer be reversed by engineering the tumor microenvironment? Seminars Cancer Biol, 2008, 18: 356-364

15 Bischof AG, Yüksel D, Mammoto T, et al. Breast cancer normalization induced by embryonic mesenchyme is mediated by extracellular matrix biglycan. Integrative Biol, 2013, 5: 1045-1056

16 Abu-Tayeh H, Weidenfeld K, Zhilin-Roth A, et al. 'Normalizing' the malignant phenotype of luminal breast cancer cells via alpha(v) beta(3)-integrin. Cell Death Dis, 2016, 7: e2491

17 Levental KR, Yu H, Kass L, et al. Matrix crosslinking forces tumor progression by enhancing integrin signaling. Cell, 2009, 139: 891906

$18 \mathrm{Hu}$ M, Chang $\mathrm{H}$, Zhang $\mathrm{H}$, et al. Mechanical adaptability of the MMP-responsive film improves the functionality of endothelial cell monolayer. Adv Healthcare Mater, 2017, 6: 1601410

19 Zhang H, Chang H, Wang L, et al. Effect of polyelectrolyte film stiffness on endothelial cells during endothelial-to-mesenchymal transition. Biomacromolecules, 2015, 16: 3584-3593

20 Audic S, Claverie JM, The significance of digital gene expression profiles. Genome Res, 1997, 7: 986-995

21 von Mering C, Huynen M, Jaeggi D, et al. STRING: A database of predicted functional associations between proteins. Nucleic Acids Res, 2003, 31: 258-261

22 Gkretsi V, Stylianopoulos T. Cell adhesion and matrix stiffness: Coordinating cancer cell invasion and metastasis. Front Oncol, 2018, 8: 145

23 Deng J, Miller SA, Wang HY, et al. $\beta$-catenin interacts with and inhibits NF- $\mathrm{kB}$ in human colon and breast cancer. Cancer Cell, 2002, 2: 323-334

24 van Kempen LCL, Coussens LM. MMP9 potentiates pulmonary metastasis formation. Cancer Cell, 2002, 2: 251-252

25 Jezierska A, Motyl T. Matrix metalloproteinase-2 involvement in breast cancer progression: A mini-review. Med Sci Monitor, 2009, 15: RA32-40

26 Mehner C, Hockla A, Miller E, et al. Tumor cell-produced matrix metalloproteinase 9 (MMP-9) drives malignant progression and metastasis of basal-like triple negative breast cancer. Oncotarget, 2014, 5: 2736-2749

27 Roskoski Jr. R. The ErbB/HER family of protein-tyrosine kinases and cancer. Pharmacol Res, 2014, 79: 34-74

28 Rau A, Lieb WS, Seifert O, et al. Inhibition of tumor cell growth and cancer stem cell expansion by a bispecific antibody targeting EGFR and HER3. Mol Cancer Ther, 2020, 19: 1474-1485

29 Klaus A, Birchmeier W. Wnt signalling and its impact on development and cancer. Nat Rev Cancer, 2008, 8: 387-398

30 Lehmann BD, Bauer JA, Chen X, et al. Identification of human triple-negative breast cancer subtypes and preclinical models for selection of targeted therapies. J Clin Invest, 2011, 121: 2750-2767

31 Lee H, Goodarzi H, Tavazoie SF, et al. TMEM2 is a SOX4-regulated gene that mediates metastatic migration and invasion in breast cancer. Cancer Res, 2016, 76: 4994-5005

32 Bilir B, Kucuk O, Moreno CS. Wnt signaling blockage inhibits cell proliferation and migration, and induces apoptosis in triple-ne- gative breast cancer cells. J Transl Med, 2013, 11: 280

33 Sun Z, Schwenzer A, Rupp T, et al. Tenascin-C promotes tumor cell migration and metastasis through integrin $\alpha 9 \beta 1$-mediated YAP inhibition. Cancer Res, 2018, 78: 950-961

34 Oskarsson T, Acharyya S, Zhang XHF, et al. Breast cancer cells produce tenascin $\mathrm{C}$ as a metastatic niche component to colonize the lungs. Nat Med, 2011, 17: 867-874

35 Gilbert PM, Havenstrite KL, Magnusson KEG, et al. Substrate elasticity regulates skeletal muscle stem cell self-renewal in culture. Science, 2010, 329: 1078-1081

36 Yang C, Tibbitt MW, Basta L, et al. Mechanical memory and dosing influence stem cell fate. Nat Mater, 2014, 13: 645-652

37 Nasrollahi S, Walter C, Loza AJ, et al. Past matrix stiffness primes epithelial cells and regulates their future collective migration through a mechanical memory. Biomaterials, 2017, 146: 146-155

38 Weaver VM, Petersen OW, Wang F, et al. Reversion of the malignant phenotype of human breast cells in three-dimensional culture and in vivo by integrin blocking antibodies. J Cell Biol, 1997, 137: 231-245

39 Bussard KM, Smith GH. Human breast cancer cells are redirected to mammary epithelial cells upon interaction with the regenerating mammary gland microenvironment in-vivo. PLoS ONE, 2012, 7: e49221

40 Kenny PA, Bissell MJ. Tumor reversion: Correction of malignant behavior by microenvironmental cues. Int J Cancer, 2003, 107: 688-695

41 Yu L, Hou Y, Xie W, et al. Self-strengthening adhesive force promotes cell mechanotransduction. Adv Mater, 2020, 32: 2006986

42 Yu L, Hou Y, Xie W, et al. Ligand diffusion enables force-independent cell adhesion via activating $\alpha 5 \beta 1$ integrin and initiating Rac and RhoA signaling. Adv Mater, 2020, 32: 2002566

43 Cheng Z, Nai S, Song S, et al. Photoinduced directional domain sliding motion in peptide hydrogels promotes ectodermal differentiation of embryonic stem cells. Sci China Mater, 2020, 63: 467478

44 Nakayama KI, Nakayama K. Ubiquitin ligases: cell-cycle control and cancer. Nat Rev Cancer, 2006, 6: 369-381

45 Mo JS, Park HW, Guan KL. The Hippo signaling pathway in stem cell biology and cancer. EMBO Rep, 2014, 15: 642-656

46 Kruiswijk F, Labuschagne CF, Vousden KH. p53 in survival, death and metabolic health: A lifeguard with a licence to kill. Nat Rev Mol Cell Biol, 2015, 16: 393-405

47 Kaur RP, Vasudeva K, Kumar R, et al. Role of p53 gene in breast cancer: focus on mutation spectrum and therapeutic strategies. Curr Pharm Des, 2018, 24: 3566-3575

48 Lundgren K, Brown M, Pineda S, et al. Effects of cyclin D1 gene amplification and protein expression on time to recurrence in postmenopausal breast cancer patients treated with anastrozole or tamoxifen: A TransATAC study. Breast Cancer Res, 2012, 14: R57

49 Rudas M, Lehnert M, Huynh A, et al. Cyclin D1 expression in breast cancer patients receiving adjuvant tamoxifen-based therapy. Clin Cancer Res, 2008, 14: 1767-1774

50 Lundberg A, Lindström LS, Li J, et al. The long-term prognostic and predictive capacity of cyclin D1 gene amplification in 2305 breast tumours. Breast Cancer Res, 2019, 21: 34

51 Fallah Y, Brundage J, Allegakoen P, et al. MYC-driven pathways in breast cancer subtypes. Biomolecules, 2017, 7: 53

52 Katoh M, Katoh M. Molecular genetics and targeted therapy of WNT-related human diseases (Review). Int J Mol Med, 2017, 40: 587-606 
53 Valenta T, Hausmann G, Basler K. The many faces and functions of $\beta$-catenin. EMBO J, 2012, 31: 2714-2736

54 Feng J, Sharma S, Rao E, et al. Stiffness heterogeneity-induced double-edged sword behaviors of carcinoma-associated fibroblasts in antitumor therapy. Sci China Mater, 2019, 62: 873-884

55 Elosegui-Artola A, Andreu I, Beedle AEM, et al. Force triggers YAP nuclear entry by regulating transport across nuclear pores. Cell, 2017, 171: 1397-1410.e14

56 Shiu JY, Aires L, Lin Z, et al. Nanopillar force measurements reveal actin-cap-mediated YAP mechanotransduction. Nat Cell Biol, 2018, 20: 262-271

57 Lee JY, Chang JK, Dominguez AA, et al. YAP-independent mechanotransduction drives breast cancer progression. Nat Commun, 2019, 10: 1848

58 Lee JY, Chaudhuri O. Regulation of breast cancer progression by extracellular matrix mechanics: Insights from 3D culture models. ACS Biomater Sci Eng, 2017, 4: 302-313

Acknowledgements This work was supported by the National Natural Science Foundation of China (21875210), the National Key Research and Development Program of China (2016YFC1102203), the Natural Key Research and Development Project of Zhejiang Province (2018C03015), Zhejiang Provincial Ten Thousand Talents Program (2018R52001), the Fundamental Research Funds for the Central Universities (2020FZZX003-01-03), and the Higher Education Discipline Innovation Project (111 Project) (B16042).

Author contributions Ren KF and Ji J conceived and supervised the project; $\mathrm{Hu} \mathrm{M}$ and Ren KF designed the experiments; Hu M and Li X carried out the experiments; Hu M wrote the manuscript. Ren KF revised the manuscript. All authors contributed to the general discussion.

Conflict of interest The authors declare that they have no conflict of interest.

Supplementary information Experimental details and supporting data are available in the online version of the paper.

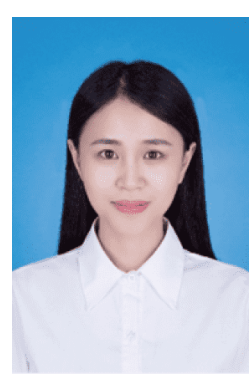

Mi $\mathbf{H u}$ is a $\mathrm{PhD}$ candidate in polymer material at Zhejiang University. Her research focuses on the construction of biocompatible polyelectrolyte films and hydrogels.

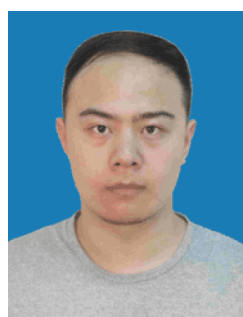

$\mathbf{X u} \mathbf{L i}$ is a $\mathrm{PhD}$ candidate in material engineering at Zhejiang University. His research focuses on the interaction between material and cells.

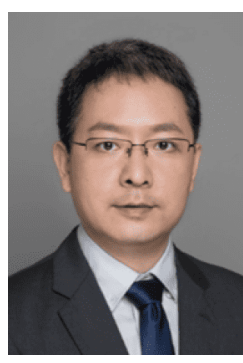

Ke-Feng Ren received his $\mathrm{PhD}$ degree from Zhejiang University in 2006. After working as a Postdoc with Prof. C. Picart in CNRS, France, he is now a full professor at Zhejiang University. $\mathrm{He}$ has co-authored over 100 peer-reviewed publications. His current research focuses on biomaterials interfaces, medical coating, cardiovascular medical devices.

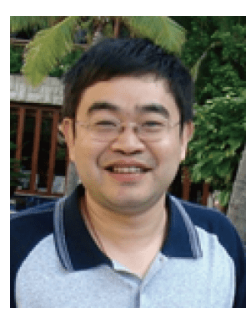

Jian Ji received his $\mathrm{PhD}$ degree from Zhejiang University in 1997. In 2010, he received the Distinguished Young Scholars Award of the National Science Foundation of China. In 2015, he was awarded as Cheung Kong Scholars by the Ministry of Education. He has co-authored over 200 peer-reviewed publications. His research focuses on interfacial phenomena for biomedical implant, tissue engineering and nanomedicine.

\section{动态硬度降低基底调控肿瘤细胞恶性的研究}

胡米 ${ }^{1 \dagger}$, 李旭 ${ }^{1 \dagger}$, 黄威嫔 ${ }^{1}$, 胡登峰 ${ }^{1}$, 任科峰 ${ }^{1,2^{*}}$, 计剑 ${ }^{1,2}$

摘要 肿瘤的发生发展伴随着细胞外基质的重塑和硬化. 目前, 尽 管细胞外基质的硬度已经成为一种强有力的肿瘤细胞行为调节器, 但基底硬度动态下降对乳腺癌细胞行为的影响却鲜有研究. 在此, 我们采用基质金属蛋白酶敏感的聚电解质多层膜进行分析, 探讨 硬度的降低对乳腺癌细胞MDA-MB-231生物学行为的影响. 结果 表明, 与在静态高硬度基底上培养的细胞相比, 在硬度动态降低的 基底上培养时, 细胞的迁移、粘附及恶性相关蛋白和基因表达水 平发生了显著变化, 肿瘤恶性降低. 根据蛋白质相互作用PPI网络 分析结果, 中枢节点基因TP53, CCND1, MYC, CTNNB1和YAP被 认为在硬度依赖性细胞行为变化中起着核心作用. 此外, 将在动态 软化基底上培养的细胞转移到高硬度材料上之后, 细胞仍保持较 弱的恶性, 表现出一定的记忆性. 